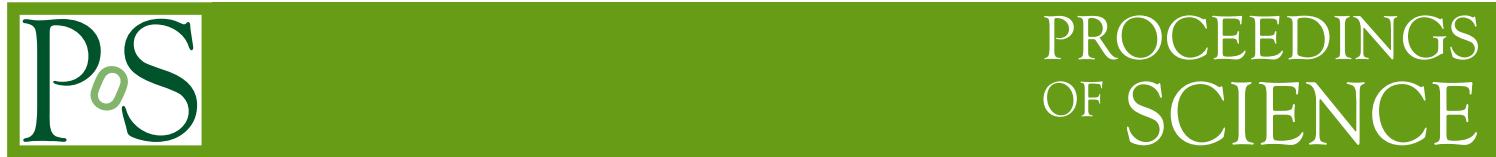

\title{
Transverse spin results from PHENIX
}

\author{
Vipuli Dharmawardane ${ }^{* \dagger}$ \\ New Mexico State University \\ E-mail: vipuliercf.rhic.bnl.gov
}

\section{Anselm Vossen \\ University of Illinois \\ E-mail: vossen@illinois.edu \\ for the PHENIX collaboration}

The Relativistic Heavy Ion Collider, the first polarized proton proton collider, has opened new opportunities to measure transverse single spin asymmetries in a new kinematic regime where perturbative QCD can be applied. PHENIX is one of the ongoing experiments studying the spin structure of the nucleon at RHIC. Recent results from the PHENIX experiment on transverse single spin asymmetries are presented, and their impact on our understanding of nucleon structure is discussed.

XVIII International Workshop on Deep-Inelastic Scattering and Related Subjects April 19 -23, 2010

Convitto della Calza, Firenze, Italy

\footnotetext{
${ }^{*}$ Speaker.

$\dagger$ I would like to thank Anselm Vossen for giving the presentation on my behalf.
} 


\section{Introduction}

Transverse single spin asymmetries (SSA) play an important role for our understanding of QCD and have attracted considerable interest among theorists and experimentalists in recent years. On the theoretical side a number of approaches within quantum chromodynamics have been proposed to explain these transverse single spin asymmetries. Among them the most widely discussed are transverse momentum dependent (TMD) parton distributions and fragmentation functions, and higher twist effects [1]. It has been demonstrated that there is a relationship between the Sivers function and the corresponding twist-3 quark-gluon and tri-gluon correlation functions. Each approach, which has it's own valid kinematic domain, is expected to describe the same physics in the overlapping kinematic region.

One of the difficulties in disentangling these spin dependent parton distribution functions and fragmentation functions is that more than one of these mechanisms contribute to the observed single spin asymmetry in most physical processes. In order to interpret the observed transverse single spin asymmetries in a meaningful way processes which are more sensitive to one particular mechanism needs to be considered. Several such processes have been identified $[2,3,4,5,6]$ and currently been measured at the PHENIX experiment at the Relativistic Heavy Ion Collider.

\section{Processes sensitive to Sivers effect}

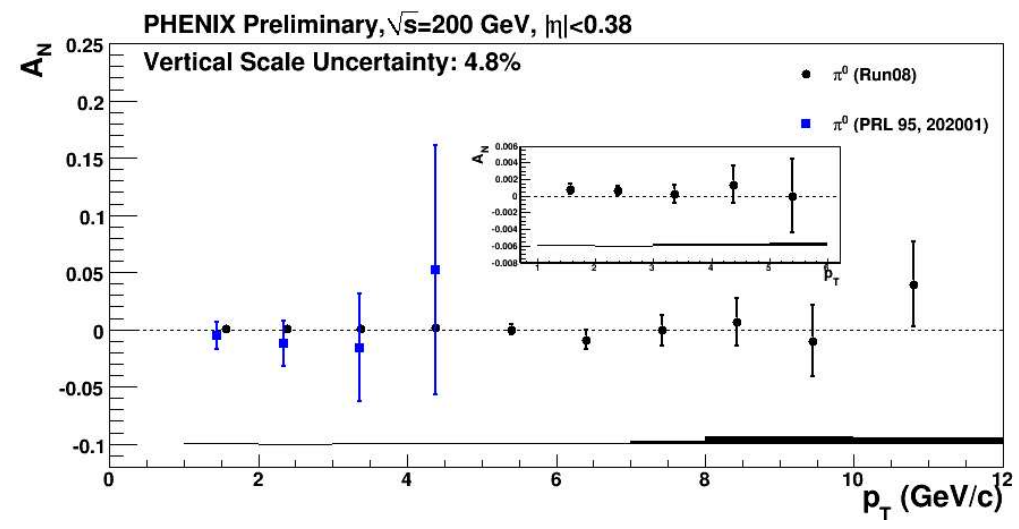

Figure 1: Transverse single spin asymmetries of neutral pions at midrapidity produced at $200 \mathrm{GeV}$.

The single transverse spin asymmetry in the process $p p \rightarrow \pi X$ is one of the most studied observables in transverse spin physics. Evaluation of different contributions to $A_{N}$ for $p p \rightarrow \pi X$ indicates that the dominant contributions are due to the Sivers and Collins mechanisms and other contributions which are relatively suppressed can be neglected [2]. The flavor decomposition of the NLO cross section for $p p \rightarrow \pi^{0} X$ shows that $A_{N}$ at large positive $x_{F}$ values are mainly driven by valence quark properties while the gluon contribution is dominant at midrapidities. PHENIX $\pi^{0}$ results on $A_{N}$ at $\sqrt{s}=200 \mathrm{GeV}$ at midrapidity published in 2005 [7] have already imposed constraints on gluon Sivers function [2] and new higher statistics results from the 2008 run shown in Figure 1 allow us to provide much tighter constraints on gluon Sivers function. As has been 

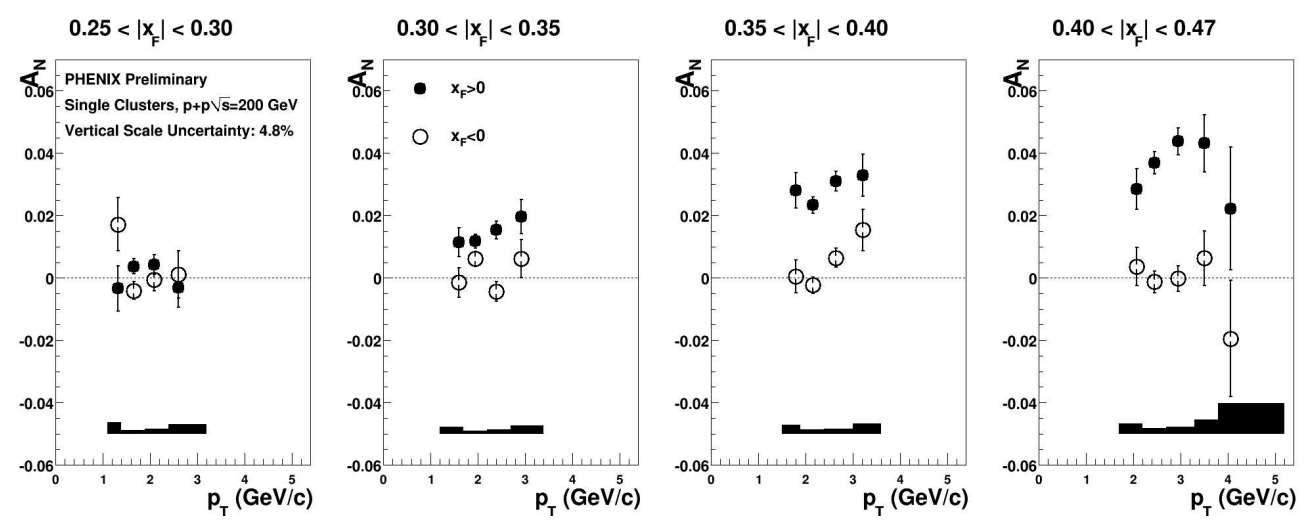

Figure 2: $P_{T}$ dependence of single spin asymmetry produced at $\sqrt{s}=200 \mathrm{GeV}$ for different $x_{F}$ bins. The asymmetry is a combination of contributions from $\pi^{0}$, direct photons and non-direct photons, while $\pi^{0}$ been the dominant contribution.

observed at much lower energies, SSA results not shown here for $\pi^{0}$ production at $\sqrt{s}=62.4 \mathrm{GeV}$ show large asymmetries in the forward region. Newer high statistics data at $200 \mathrm{GeV}$ shown in Figure 2 allow studies of the $P_{T}$ dependence of the asymmetry in addition to the $x_{F}$ dependence. The magnitude of the asymmetry appears to increase with increasing $P_{T}$, contrary to predictions made by phenomenological studies that involve twist-3 parton correlation functions. However, it is important to emphasize that asymmetries shown are not purely due to $\pi^{0} \mathrm{~s}$, and currently simulations are underway to correct for background effects.
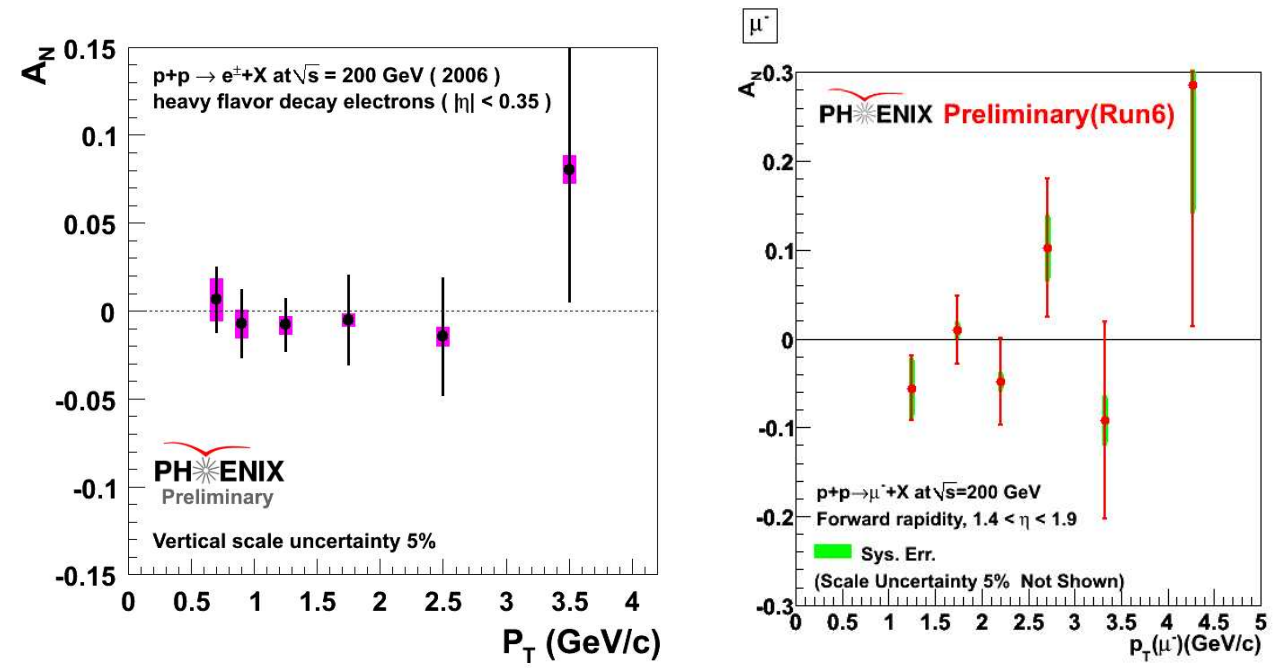

Figure 3: Heavy flavor contribution to the transverse single spin asymmetry at midrapidity as a function of electron $P_{T}$ (left) and at forward rapidities as a function of muon $P_{T}$ (right).

Figure 3 presents the measured heavy flavor contribution to the transverse single spin asymmetry at midrapidity $(|\eta|<0.35)$ and at forward rapidities $(1.4<|\eta|<1.9)$ at $\sqrt{s}=200 \mathrm{GeV}$. In a recent calculation, QCD collinear factorization approach has been employed in predicting single 
transverse-spin asymmetry for open charm production in polarized pp collisions at RHIC [4]. In addition, predictions for SSA in $D$ meson production have also been made within the TMD approach, and it has been argued that the observed transverse single spin asymmetry for this process can arise only due to the Sivers mechanism [3]. At RHIC kinematics the dominant contribution to the cross-section and the spin asymmetry for the process $p p \rightarrow D X$ is due to the gluon fusion process. Therefore these new results will allow us to cleanly access gluon Sivers distributions. The midrapidity results provide evidence of a gluon Sivers distribution function consistent with zero, and the error bars prevent us from making a clear conclusion for forward rapidity results. Further, these new results can be used in phenomenological analyses to constraint model parameters and determine quantitative bounds on trigluon correlations.

Presence of a Sivers-type correlation is expected to produce a "left-right" imbalance in intrinsic transverse momentum $k_{T}$ of partons. This "left-right" imbalance in $k_{T}$ in turn will affect the azimuthal angle distribution and hence the polarized cross section of jets nearly opposite to the first jet, and will allow us to directly access the Sivers function via transverse single spin asymmetry for this process [5]. Further, it has been shown that it is possible to access the same physics by studying SSA for two hadrons back-to-back in azimuth instead of di-jets, and has been measured at PHENIX and is shown in Figure 4. However, in the fragmentation process the Collins effect may also contribute to the measured di-hadron asymmetry. If the contribution due to the Collins effect is negligible, the small asymmetry at mid rapidity could be attributed to the cancellation of contributions due to initial and final-state interactions of partons. There are plans to expand this measurement into different combinations of rapidities, which include mid- and forward-rapidities, of the two correlating hadrons.

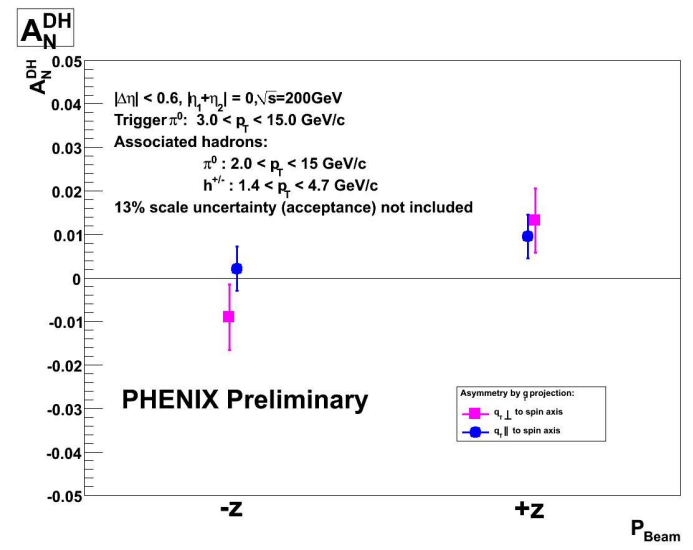

Figure 4: SSA in azimuthal correlations of di-hadrons produced back-to-back at midrapidity. The pink filled squares correspond to the Sivers asymmetry and the blue circles correspond to a controlled measurement which is expected to produce zero Sivers asymmetry." $+Z$ " and "-Z" correspond to the two RHIC beams.

\section{Processes sensitive to transversity}

Figure 5 shows the single spin asymmetry $A_{U T}^{\sin \phi}$ for hadron pairs produced within the same jet cone, which contains a product of the transversity distribution and a spin-dependent di-hadron 
fragmentation function known as the interference fragmentation function (IFF) [6]. With new data on the IFF becoming available from other experiments [8], it is possible to disentangle transversity distribution from these results. Analysis of data taken at forward kinematics, where valence quarks are predicted to make a non-zero contribution to the asymmetry, is currently underway.

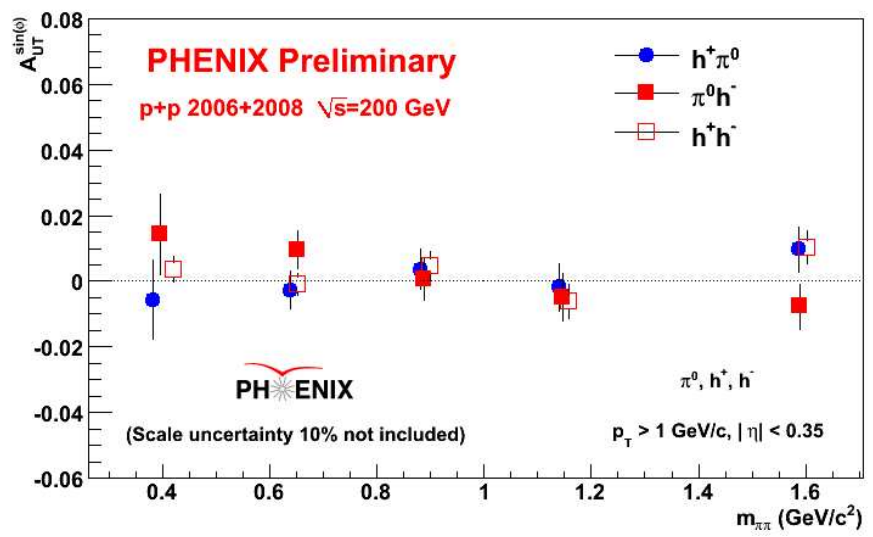

Figure 5: Single spin asymmetries for hadron pairs produced within the same jet cone as a function of invariant mass of the hadron pair at midrapidity.

\section{Summary}

RHIC has opened new opportunities to study transverse spin in a new energy regime. While much remains to be learned, new transverse single spin asymmetry results from the PHENIX experiment at RHIC are contributing to enrich our understanding of the structure of the nucleon. In addition, future detector upgrades at PHENIX and higher beam energies of RHIC offer a wealth of new opportunities.

\section{References}

[1] D. W. Sivers, Phys. Rev. D 41, 83 (1990); J. C. Collins, Nucl. Phys. B 396, 161 (1993); J. w. Qiu and G. Sterman, Phys. Rev. Lett. 67, 2264 (1991).

[2] M. Anselmino, M. Boglione, U. D’ Alesio, E. Leader, S. Melis and F. Murgia, Phys. Rev. D 73, 014020 (2006); M. Anselmino, U. D’Alesio, S. Melis and F. Murgia, Phys. Rev. D 74, 094011 (2006).

[3] M. Anselmino, M. Boglione, U. D'Alesio, E. Leader and F. Murgia, Phys. Rev. D 70, 074025 (2004).

[4] Z. B. Kang, J. W. Qiu, W. Vogelsang and F. Yuan, Phys. Rev. D 78, 114013 (2008).

[5] D. Boer and W. Vogelsang, Phys. Rev. D 69, 094025 (2004); C. J. Bomhof, P. J. Mulders, W. Vogelsang and F. Yuan, Phys. Rev. D 75, 074019 (2007).

[6] A. Bacchetta and M. Radici, Phys. Rev. D 74, 114007 (2006).

[7] S. S. Adler et al. [PHENIX Collaboration], Phys. Rev. Lett. 95, 202001 (2005).

[8] A. Vossen, R. Seidl, M. Grosse-Perdekamp, M. Leitgab, A. Ogawa and K. Boyle, arXiv:0912.0353 [hep-ex]. 